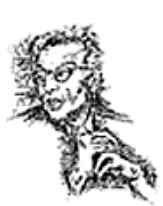

\title{
ON PRODUCT DIFFERENCE FIBONACCI IDENTITIES
}

\author{
R. S. Melham \\ Department of Mathematical Sciences, University of Technology, Sydney, Australia \\ ray.melham@uts.edu. au
}

Received: 3/31/10, Accepted: 12/9/10, Published: 1/31/11

\begin{abstract}
Simson's identity is a well-known Fibonacci identity in which the difference of certain order 2 products has a particularly pleasing form. Other old and beautiful identities of a similar nature are attributed to Catalan, Gelin and Cesàro, and Tagiuri. Catalan's identity can be described as a family of product difference Fibonacci identities of order 2 with 1 parameter. In Section 2 of this paper we present four families of product difference Fibonacci identities that involve higher order products. Being self-dual, each of these families may be regarded as a higher order analogue of Catalan's identity. We also state two conjectures that give the form of similar families of arbitrary order. In the final section we give other interesting product difference Fibonacci identities.
\end{abstract}

\section{Introduction}

The Fibonacci numbers are defined, for all integers $n$, by

$$
F_{n}=F_{n-1}+F_{n-2}, \quad F_{0}=0, \quad F_{1}=1 \text {. }
$$

The Lucas numbers are defined, for all integers $n$, by

$$
L_{n}=L_{n-1}+L_{n-2}, \quad L_{0}=2, \quad L_{1}=1
$$

By a Product Difference Fibonacci Identity (PDFI) of order $s$ we mean an identity of the form

$$
\prod_{i=1}^{s} F_{n+a_{i}}-\prod_{i=1}^{s} F_{n+b_{i}}=D_{n}\left(a_{i}, b_{i} ; s\right)=D_{n},
$$

where $s \geq 1$, and $a_{i}$ and $b_{i}$ are specified integers. This notation and terminology was introduced by Fairgrieve and Gould [5].

Two well-known and attractive PDFIs of orders 2 and 4, respectively, are

$$
F_{n-1} F_{n+1}-F_{n}^{2}=(-1)^{n},
$$


and

$$
F_{n-2} F_{n-1} F_{n+1} F_{n+2}-F_{n}^{4}=-1 .
$$

According to Dickson [3, page 393], (2) was discovered by Robert Simson in 1753. Again, according to Dickson [3, page 401], (3) was stated by E. Gelin and proved by E. Cesàro. Indeed, our discovery of (21) and (22) was motivated by the beauty of $(3)$.

The following generalization of $(2)$ is due to Catalan [1]:

$$
F_{n-a} F_{n+a}-F_{n}^{2}=(-1)^{n+a+1} F_{a}^{2} .
$$

Morgado [7] used (4) to give an alternative proof of (3). More generally there is

$$
F_{n+a} F_{n+b}-F_{n} F_{n+a+b}=(-1)^{n} F_{a} F_{b},
$$

which Dickson [3, page 404] attributes to A. Tagiuri in 1901. Everman, Danese, and Venkannayah [4] rediscovered (5) in 1960, submitting it as a problem for readers of The American Mathematical Monthly.

In order to bridge the gap between (2) and (3), Melham [6] offered the following PDFI of order 3:

$$
F_{n+1} F_{n+2} F_{n+6}-F_{n+3}^{3}=(-1)^{n} F_{n} .
$$

Melham also noticed the companion formula

$$
F_{n} F_{n+4} F_{n+5}-F_{n+3}^{3}=(-1)^{n+1} F_{n+6},
$$

but gave it little significance since it can be obtained from (6). More precisely, in (6) we replace $n$ with $-n$, and use the fact that

$$
F_{-n}=(-1)^{n+1} F_{n}
$$

to obtain $(7)$.

Following up on Melham's contribution, Fairgrieve and Gould [5] chose to write (6) and (7) as

$$
F_{n-2} F_{n-1} F_{n+3}-F_{n}^{3}=(-1)^{n-1} F_{n-3},
$$

and

$$
F_{n+2} F_{n+1} F_{n-3}-F_{n}^{3}=(-1)^{n} F_{n+3},
$$

respectively. They then called (9) and (10) dual identities since, with the use of (8), each identity can be obtained from the other by the replacement of $n$ with $-n$. Fairgrieve and Gould also discovered

$$
F_{n-2} F_{n+1}^{2}-F_{n}^{3}=(-1)^{n-1} F_{n-1}
$$

and

$$
F_{n+2} F_{n-1}^{2}-F_{n}^{3}=(-1)^{n} F_{n+1},
$$


another pair of dual identities of order 3 that Melham had missed.

Fairgrieve and Gould called each of (2) and (3) self-dual identities, since the dual of each is itself. Interestingly, (4) is self-dual, but (5) is not self-dual. Both, however, are certainly beautiful.

Furthermore, inspired by (2) and (3), Fairgrieve and Gould considered the product differences

$$
\prod_{i=1}^{s} F_{n-i} F_{n+i}-F_{n}^{2 s}
$$

for $s=3,4,5, \ldots$. They showed how to produce a self-dual PDFI of order $2 s$ for each $s=3,4,5, \ldots$. Each such identity can therefore be regarded as a higher order analogue of (2) and (3). They produced no self-dual PDFI of odd order, and we have not cited such an identity in the literature.

Let us agree to designate Catalan's identity as a family of PDFIs of order 2 with 1 parameter. Therefore, we say that Tagiuri's identity is a family of PDFIs of order 2 with 2 parameters. In Section 2 , for each $3 \leq s \leq 6$, we present a family of PDFIs of order $s$ with $s-1$ parameters. Furthermore, like Catalan's identity, each of these four families is self-dual. We therefore regard each of these families as a higher order analogue of Catalan's identity.

In Section 2 we state our main results, and in Section 3 we indicate a method of proof. In Section 4, we state two conjectures pertaining to higher order analogues of

our main results. Finally, in Section 5, we give other product difference Fibonacci identities that we feel are interesting.

\section{The Main Results}

Theorem 1. If $a$ and $b$ are integers, then

$$
F_{n+a+b} F_{n-a} F_{n-b}-F_{n-a-b} F_{n+a} F_{n+b}=(-1)^{n+a+b} F_{a} F_{b} F_{a+b} L_{n} .
$$

Theorem 2. If $a, b$, and $c$ are integers, then

$$
\begin{array}{r}
F_{n+a+b+c} F_{n-a} F_{n-b} F_{n-c}-F_{n-a-b-c} F_{n+a} F_{n+b} F_{n+c} \\
=(-1)^{n+a+b+c} F_{a+b} F_{a+c} F_{b+c} F_{2 n} .
\end{array}
$$

We have discovered generalizations of Theorems 1 and 2, and these generalizations are given in Theorems 5 and 6 , respectively. 
So that we can state the next theorem succinctly, we introduce some notation. Define

$$
\begin{array}{r}
\Delta(n ; a, b, c, d)=F_{n+a+b+c+d} F_{n-a} F_{n-b} F_{n-c} F_{n-d} \\
\quad-F_{n-a-b-c-d} F_{n+a} F_{n+b} F_{n+c} F_{n+d} .
\end{array}
$$

Then we have

Theorem 3. If $a, b, c$, and $d$ are integers, then

$$
\begin{aligned}
\Delta(n ; a, b, c, d)= & (-1)^{n} L_{n}\left\{\Delta(-1 ; a, b, c, d) F_{n}^{2}\right. \\
& \left.+(-1)^{a+b+c+d} F_{a} F_{b} F_{c} F_{d} F_{a+b+c+d} F_{n-1} F_{n+1}\right\} .
\end{aligned}
$$

To state the next theorem we use the notation in (15) with an extra parameter, and with the obvious meaning.

Theorem 4. If $a, b, c, d$, and $e$ are integers, then

$$
\begin{aligned}
\Delta(n ; a, b, c, d, e)= & (-1)^{n} F_{2 n}\left\{\Delta(-1 ; a, b, c, d, e) F_{n}^{2}\right. \\
& \left.-\frac{1}{6}[\Delta(-2 ; a, b, c, d, e)+3 \Delta(-1 ; a, b, c, d, e)] F_{n-1} F_{n+1}\right\} .
\end{aligned}
$$

\section{The Proofs}

We have managed to prove the theorems in the previous section with the verification theorem of Dresel [2, page 171]. To illustrate, we prove Theorem 4.

In the terminology of Dresel, the identity in Theorem 4 is homogeneous of degree 6 in the variable $n$. As Dresel explains, since $(-1)^{n}=(\alpha \beta)^{n}$, where $\alpha$ and $\beta$ are the roots of $x^{2}-x-1=0$, then $(-1)^{n}$ is of degree 2 in the variable $n$. Furthermore, since, for instance, $F_{2-a}=F_{-(a-2)}=(-1)^{a-1} F_{a-2}$, then $F_{2-a}$ is of degree 3 in the variable $a$.

Since the identity in Theorem 4 is homogeneous of degree 6 in the variable $n$, to prove it with the verification theorem of Dresel we need only verify its validity for seven distinct values of $n$. Accordingly, we write down the cases that correspond to $n=1,2,3,4,5,6$, and 7 . We are required to prove each of these seven cases. Now, each of these seven cases is an identity that is homogeneous of degree 4 in the variable $a$. Therefore, to prove any one of these seven cases, we need only verify its validity for five distinct values of $a$; say $a=1,2,3,4$, and 5 . Thus far, we have thirty-five identities in the variables $b, c, d$, and $e$ that we are required to prove. 
Continuing in the same manner, we are ultimately required to verify the identity stated in Theorem 4 for $7 \times 5 \times 5 \times 5 \times 5 \times 5$ distinct ordered 6 -tuples $(n, a, b, c, d, e)$. With six nested "For" loops, we managed to perform these verifications, and thereby complete the proof of Theorem 4 in a matter of seconds with the use of the computer algebra system Mathematica 6.0 .

In the final paragraph of the introduction we remarked that the identities in Theorems 1-4 are self-dual. This is easily seen with the use of (8) together with the fact that $L_{-n}=(-1)^{n} L_{n}$.

\section{Families of Self-Dual PDFIs Order 7 and Higher}

We have continued our investigation into higher order analogues of Theorems 1-4. In this section we state our observations as conjectures that set forth higher order families of PDFIs that are self-dual. Once again we employ the same notation used in Theorems 3 and 4.

Our first conjecture deals with PDFIs of order $7,9,11, \ldots$

Conjecture 1. For $k \geq 2$ let $a_{1}, \ldots, a_{2 k+2}$ be arbitrary integers. Then

$$
\Delta\left(n ; a_{1}, \ldots, a_{2 k+2}\right)=(-1)^{n+a_{1}+\cdots+a_{2 k+2}} L_{n}\left(c_{2 k} F_{n}^{2 k}+\sum_{i=1}^{2 k-1} c_{2 k-i} F_{n-1}^{2 k-i} F_{n+1}^{i}\right)
$$

in which $c_{2 k-i}=c_{i}$ for $i=1, \ldots, k-1$.

Our second conjecture deals with PDFIs of order $8,10,12, \ldots$.

Conjecture 2. For $k \geq 2$ let $a_{1}, \ldots, a_{2 k+3}$ be arbitrary integers. Then

$$
\Delta\left(n ; a_{1}, \ldots, a_{2 k+3}\right)=(-1)^{n+a_{1}+\cdots+a_{2 k+3}} F_{2 n}\left(c_{2 k} F_{n}^{2 k}+\sum_{i=1}^{2 k-1} c_{2 k-i} F_{n-1}^{2 k-i} F_{n+1}^{i}\right),
$$

in which $c_{2 k-i}=c_{i}$ for $i=1, \ldots, k-1$.

In both of these conjectures the coefficients $c_{i}$ are expressible in terms of $\Delta$ with the appropriate arguments. Furthermore, we can find the $c_{i}$ by substituting various values of $n$, and solving the resulting system of linear equations. Indeed, because of the symmetry in the $c_{i}$, this task can be accomplished with $k+1$ different values of $n$.

To illustrate, we consider Conjecture 1 for the case $k=2$ and find the coefficients $c_{i}$. Furthermore, we prove this case of Conjecture 1 so that this case becomes a theorem. Since $c_{1}=c_{3}$, we require only three equations to solve for $c_{1}, c_{2}, c_{3}$, and $c_{4}$. We form these three equations by substituting $n=0, n=1$, and $n=2$, and solve these equations to obtain 


$$
\begin{aligned}
& c_{1}=-\frac{1}{6}(-1)^{a_{1}+\cdots+a_{6}}\left[6 \Delta\left(0 ; a_{1}, \ldots, a_{6}\right)-3 \Delta\left(1 ; a_{1}, \ldots, a_{6}\right)-\Delta\left(2 ; a_{1}, \ldots, a_{6}\right)\right], \\
& c_{2}=\frac{1}{6}(-1)^{a_{1}+\cdots+a_{6}}\left[15 \Delta\left(0 ; a_{1}, \ldots, a_{6}\right)-6 \Delta\left(1 ; a_{1}, \ldots, a_{6}\right)-2 \Delta\left(2 ; a_{1}, \ldots, a_{6}\right)\right], \\
& c_{3}=-\frac{1}{6}(-1)^{a_{1}+\cdots+a_{6}}\left[6 \Delta\left(0 ; a_{1}, \ldots, a_{6}\right)-3 \Delta\left(1 ; a_{1}, \ldots, a_{6}\right)-\Delta\left(2 ; a_{1}, \ldots, a_{6}\right)\right], \\
& c_{4}=-(-1)^{a_{1}+\cdots+a_{6}} \Delta\left(1 ; a_{1}, \ldots, a_{6}\right) .
\end{aligned}
$$

Finally, in the same manner as for the proof of Theorem 4, we perform verifications for $8 \times 5 \times 5 \times 5 \times 5 \times 5 \times 5$ appropriately chosen ordered 7 -tuples $\left(n, a_{1}, \ldots, a_{6}\right)$. We can verify that we have indeed completed these verifications, thus establishing the truth of Conjecture 1 for $k=2$.

\section{Other PDFIs of Interest}

In the course of our research, we discovered other interesting PDFIs that are worthy of mention.

The five identities that follow are of order 3 , and none is self-dual. To conserve space, we give only one identity in each dual pair.

$$
\begin{gathered}
F_{n-3} F_{n+1}^{2}-F_{n-2}^{2} F_{n+3}=4(-1)^{n} F_{n}, \\
F_{n-1}^{2} F_{n+3}-F_{n}^{2} F_{n+1}=(-1)^{n} L_{n}, \\
F_{n-1} F_{n+1}^{2}-F_{n-2} F_{n} F_{n+3}=(-1)^{n} L_{n+1}, \\
F_{n-5} F_{n+2}^{2}-F_{n-1}^{2} F_{n+1}=4(-1)^{n} L_{n+1}, \\
F_{n-1} F_{n}^{2}-F_{n-4} F_{n+1} F_{n+2}=(-1)^{n} L_{n+2} .
\end{gathered}
$$

The two identities that follow are of order 4, and both are self-dual. By any measure they are certainly attractive.

$$
\begin{gathered}
F_{n-1}^{2} F_{n+1}^{2}-F_{n-2}^{2} F_{n+2}^{2}=4(-1)^{n} F_{n}^{2}, \\
F_{n-3} F_{n-1} F_{n+1} F_{n+3}-F_{n}^{4}=(-1)^{n} L_{n}^{2} .
\end{gathered}
$$

Each of (16)-(20) is homogeneous of degree 3 in the variable $n$. Accordingly, to prove (16)-(20), we need only verify the validity of each for four distinct values of $n$. Likewise, to prove (21) and (22), we need only verify the validity of each for five distinct values of $n$.

Alternatively, we can prove each of (16)-(22) by expressing the left and right sides as polynomials in $F_{n}$ and $F_{n+1}$, using Simson's identity to substitute $F_{n-1} F_{n+1}-F_{n}^{2}$ 
for $(-1)^{n}$. We leave the details to the reader. Identity (21) also follows from Simson's identity and Catalan's identity upon factorization of the left side.

In our next theorem we give a family of PDFIs of order 3 with 3 parameters. For $c=0$ this family reduces to the family given in Theorem 1 , otherwise the identities in (23) are not self-dual. In Theorem $5,(a, b, c)=(1,1,-1)$ yields (16), while $(a, b, c)=(-1,-1,1)$ yields the dual of $(16)$.

Theorem 5. If $a, b$, and $c$ are integers, then

$$
\begin{aligned}
& F_{n+a+b-c} F_{n-a+c} F_{n-b+c}-F_{n-a-b+c} F_{n+a} F_{n+b} \\
& \quad=(-1)^{n+a+b+c} F_{a+b-c}\left(F_{c} F_{n+a+b-c}+(-1)^{c} F_{a-c} F_{b-c} L_{n}\right) .
\end{aligned}
$$

In our final theorem we present a pleasing family of PDFIs of order 4 with 4 parameters. For $d=0$ the family of identities in (24) reduces to the family given in Theorem 2, otherwise the identities in (24) are not self-dual.

Theorem 6. If $a, b, c$, and $d$ are integers, then

$$
\begin{array}{r}
F_{n+a+b+c-d} F_{n-a+d} F_{n-b+d} F_{n-c+d}-F_{n-a-b-c+2 d} F_{n+a} F_{n+b} F_{n+c} \\
=(-1)^{n+a+b+c} F_{a+b-d} F_{a+c-d} F_{b+c-d} F_{2 n+d} .
\end{array}
$$

The proofs of Theorems 5 and 6 follow along the same lines described in Section 3. Note that identity (24) is homogeneous of degree 4 in the variables $a, b, c$, and $n$. The product on the right of (24) is of degree 10 in the variable $d$. We therefore insert $(-1)^{2 d}$ in the first product on the left, and $(-1)^{4 d}$ in the second product on the left. This makes (24) homogeneous of degree 10 in the variable $d$. Therefore, to prove (24) with Dresel's verification theorem, we are required to verify it for $5 \times 5 \times 5 \times 5 \times 11$ distinct ordered 5 -tuples $(n, a, b, c, d)$. Once again, we performed these verifications with the use Mathematica 6.0 , thereby proving Theorem 6 .

A little care is needed in the proof of Theorem 5. It is easy to see that (23) is homogeneous of degree 3 in $n$, and homogeneous of degree 4 in $a$ and also in $b$. For the variable $c$ we need to insert various powers of $(-1)^{c}$ in the appropriate places to see that (23) is homogeneous of degree 13 in $c$. The proof of Theorem 5 then follows by Dresel's verification theorem. 


\section{References}

[1] E. Catalan, Sur la Serié de Lamé, Mémoires de la Société Royale des Sciences de Liège (Series 2), 13 (1886), 319-321.

[2] L. A. G. Dresel, Transformations of Fibonacci-Lucas Identities, Applications of Fibonacci Numbers, 5 (1993), 169-184.

[3] L. E. Dickson, History of the Theory of Numbers, Vol 1, Chelsea, 1966.

[4] D. Everman, A. Danese, and Venkannayah, Problem E1396, The American Mathematical Monthly, 67 (1960), 81-82.

[5] S. Fairgrieve and H. W. Gould, Product Difference Fibonacci Identities of Simson, GelinCesàro, Tagiuri and Generalizations, The Fibonacci Quarterly, 43 (2005), 137-141.

[6] R. S. Melham, A Fibonacci Identity in the Spirit of Simson and Gelin-Cesàro, The Fibonacci Quarterly, 41 (2003), 142-143.

[7] J. Morgado, Some Remarks on an Identity of Catalan Concerning the Fibonacci Numbers, Portugaliae Mathematica, 39 (1980), 341-348. 